\title{
Evaluating the potential of cubosomal nanoparticles for oral delivery of amphotericin B in treating fungal infection
}

This article was published in the following Dove Press journal:

International Journal of Nanomedicine

6 January 2014

Number of times this article has been viewed

\author{
Zhiwen Yangl,3 \\ Meiwan Chen ${ }^{2}$ \\ Muhua Yang' \\ Jian Chen' \\ Weijun Fang' \\ Ping $X u^{\prime}$ \\ 'Department of Pharmacy, Songjiang \\ Hospital Affiliated The First People's \\ Hospital, Shanghai Jiao Tong University, \\ Shanghai, ${ }^{2}$ State Key Laboratory of \\ Quality Research in Chinese Medicine, \\ Institute of Chinese Medical Sciences, \\ University of Macau, Macau, ${ }^{3}$ Shanghai \\ Songjiang Hospital Affiliated Nanjing \\ Medical University, Nanjing, People's \\ Republic of China
}

\begin{abstract}
The oral administration of amphotericin B (AmB) has a major drawback of poor bioavailability. The aim of this study was to investigate the potential of glyceryl monoolein (GMO) cubosomes as lipid nanocarriers to improve the oral efficacy of AmB. Antifungal efficacy was determined in vivo in rats after oral administration, to investigate its therapeutic use. The human colon adenocarcinoma cell line (Caco-2) was used in vitro to evaluate transport across a model of the intestinal barrier. In vivo antifungal results showed that AmB, loaded in GMO cubosomes, could significantly enhance oral efficacy, compared against Fungizone ${ }^{\circledR}$, and that during a 2 day course of dosage $10 \mathrm{mg} / \mathrm{kg}$ the drug reached effective therapeutic concentrations in renal tissue for treating fungal infections. In the Caco-2 transport studies, GMO cubosomes resulted in a significantly larger amount of AmB being transported into Caco- 2 cells, via both clathrin- and caveolae-mediated endocytosis, but not macropinocytosis. These results suggest that GMO cubosomes, as lipid nanovectors, could facilitate the oral delivery of AmB.
\end{abstract}

Keywords: glyceryl monoolein cubosomes, oral delivery, amphotericin B, antifungal activity, absorption mechanism

\section{Introduction}

Until now, its limited properties of solubility and permeability have made it impossible to develop a practical oral administration for amphotericin B (AmB); no AmB product has been successfully developed into an oral formulation for the market. Different research groups have incorporated AmB into various carrier systems for oral delivery, including carbon nanotubes, ${ }^{1}$ polymer lipid hybrid nanoparticles, ${ }^{2}$ polymeric nanoparticles, ${ }^{3}$ nanosuspensions, ${ }^{4}$ solid lipid nanoparticles, ${ }^{5}$ phytantriol (PHY) cubosomes, ${ }^{6,7}$ self-emulsifying drug delivery systems (SEDDS), ${ }^{8,9}$ and cochleates. ${ }^{10}$ The two oral lipid-based formulations draw attention to their ability to enhance oral bioavailability of $\mathrm{AmB}$, which leads to improved fungicidal efficacy after oral administration. The AmB cochleate formulation is undergoing clinical trials for its oral delivery by Biodelivery Sciences International Inc (Raleigh, NC, USA). ${ }^{11}$ Emulsion, the other lipid-based AmB formulation, is currently being developed for oral administration by iCo Therapeutics Inc (Vancouver, BC, Canada). ${ }^{11}$ However, cochleates are composed of the expensive dioleoyl phosphatidylserine (DOPS), and emulsions are unstable because of their physical characteristics.

Interestingly, most studies have focused on glyceryl monoolein (GMO) as a liquid crystal-forming lipid for enhancing oral bioavailability. ${ }^{12}$ The unique liquid crystalline structure of cubosomes could provide similar protection to cochleates, when exposed to harsh environmental conditions or enzymes in the gastrointestinal tract. ${ }^{13}$
Songjiang Hospital Affiliated the First People's Hospital, Shanghai Jiao

Tong University, Shanghai, 201600,

People's Republic of China

Tel +862I 67720472

Email luozonghua2012@126.com submit your manuscript $\mid$ www.dovepress.com

Dovepress

http://dx.doi.org//0.2147/IJN.S54967
International Journal of Nanomedicine 2014:9 327-336

(c) (i) (5) 2014 Yang et al. This work is published by Dove Medical Press Limited, and licensed under Creative Commons Attribution - Non Commercial (unported, v3.0) License. The full terms of the License are available at http://creativecommons.org/licenses/by-nd/3.0/. Non-commercial uses of the work are permitted without any further permission Love Medical Press Limited, provided the work is properly attributed. Permissions beyond the scope of the License are administered by Dove Medical Press Limited. Information on how to request permission may be found at: http://www.dovepress.com/permissions.php 
In addition, the GMO-lipid composition of cubosomes could increase gastrointestinal absorption of AmB, just as with GMO micelle, GMO solution, and emulsion-enhanced oral AmB bioavailability. ${ }^{8}$ GMO is a safe and cheap lipid excipient, and is regarded as the better alternative for oral administration, compared against other lipid excipients (PHY, DOPS, etc)..$^{14}$

Therefore, the purpose of this study was to investigate the GMO cubosomal formulation for AmB oral administration, focusing on antifungal efficacy in vivo and cellular mechanisms of a human colon adenocarcinoma cell line (Caco-2).

\section{Materials and methods}

\section{Materials}

AmB was obtained from North China Pharmaceutical Group Corporation (Shenyang, People's Republic of China), and Fungizone ${ }^{\circledR}$ was a gift sample from the same company. GMO (monoolein) DIMONANR MO 90/D (GMO content >99\%) was kindly donated by Danisco Cultor (Grindsted, Denmark). Poloxamer $407\left(\mathrm{PEO}_{98} \mathrm{POP}_{67} \mathrm{PEO}_{98}\right)$ was a gift from BASF (Ludwigshafen, Germany). Acetonitrile (HPLC grade) was purchased from SK Chemicals (Seoul, Korea). Milli-Q grade water purified through a Millipore system (ELGA LabWater, Sartorius, UK) was used throughout the present study.

\section{Preparation of AmB-loaded cubosomes}

AmB-loaded cubosomes were prepared according to a previously-described method. ${ }^{7}$ GMO and P407, at a ratio of $9: 1$ (by weight), were melted at $60^{\circ} \mathrm{C}$ in a hot water bath until homogenous. Then, $2 \mathrm{~mL}$ of deionized water, containing AmB, was added gradually and vortex mixed for 1 minute to achieve a homogenous state. The resulting milky, coarse dispersions were homogenized using a high pressure homogenizer (Avestin Em-C3, Ottawa, ON, Canada), at certain high pressures and cycles, to obtain an opalescent dispersion of the cubosomes.

\section{Characterization of AmB-loaded cubosomes}

\section{Particle size measurements}

The mean z-average diameter and polydispersity indices of the cubosomes were measured by photon correlation spectroscopy using a Zetasizer Nano ZS90 (Malvern Instruments, Malvern, UK).

\section{Encapsulation efficiency}

The percentage of drug incorporated into cubosomes was determined by a centrifugal ultrafiltration method. ${ }^{6}$
Experiments were performed using YM-100 centrifugal tubes from Millipore Corporation (Billerica, MA, USA). After centrifuging the cubosome dispersion at 15,000 rpm for 30 minutes, the free AmB with filtrate was removed through the bottom of the reservoir of the centricon, with a certain pore size. Then, the retentate in the reservoir was diluted with methanol, and the entrapped AmB content was analyzed via high performance liquid chromatography (HPLC).

\section{UV absorption spectra}

UV spectroscopic studies were carried out to monitor the molecular state of AmB in cubosomal formulation. The sample from AmB deoxycholate, AmB-loaded cubosomes, and a mixture of AmB deoxycholate and blank cubosomes were respectively diluted in water to reach a final drug concentration of $2 \mathrm{mg} / \mathrm{mL}$, and were then investigated using ultraviolent visible spectroscopy, with $\lambda=250-500 \mathrm{~nm}$. All spectra were recorded against a blank, consisting of AmB-free preparations, in the reference beam, at $25^{\circ} \mathrm{C}$.

\section{Aggregation state in simulated gastric (SGF) and fasted state simulated intestinal fluid (FaSSIF)}

The stability experiment was divided into three groups: AmB deoxycholate, AmB-loaded cubosomes, and a mixture of AmB deoxycholate and blank cubosomes. Samples containing $2 \mathrm{mg} / \mathrm{mL}$ of AmB were added to SGF or FaSSIF, at the dilution rate of 1:10 (by volume). After 120 minutes of incubation at room temperature, the samples were characterized by optical microscopy (Nikon Diaphot, Nikon, Tokyo, Japan).

\section{Caco- 2 cell culture}

Caco-2 was obtained from the American Type Culture Collection (Manassas, VA, USA). Caco-2 cells were seeded inside Transwell ${ }^{\circledR}$ cell culture chambers (Corning Costar, Cambridge, MA, USA) at a density of $0.1 \times 10^{6}$ cells per insert. After 18-21 days in culture, cell monolayers with transepithelial electrical resistance (TEER) values greater than 250 $\Omega / \mathrm{cm}^{2}$ were carried through to the following assays.

\section{Transport across Caco-2 cells}

For the apical to basolateral (A-B) transport study, ${ }^{15} 0.5 \mathrm{~mL}$ of AmB-loaded cubosomes, diluted with transport medium, was added to the apical side. For the basolateral to apical (B-A) transport study, ${ }^{15}$ AmB-loaded cubosomes were placed into the basolateral side. At predetermined time intervals, $0.2 \mathrm{~mL}$ of medium was taken, and was replaced with fresh 
medium. The concentration of $\mathrm{AmB}$ was determined by HPLC analysis, and the apparent permeability coefficient (Papp) was calculated according to the following equation,

$$
\text { Papp }=\mathrm{dQ} / \mathrm{dt} \times 1 / \mathrm{A} \cdot \mathrm{C}_{0}
$$

where $\mathrm{dQ} / \mathrm{dt}$ is the rate of drug appearance on the basolateral side $(\mu \mathrm{g} / \mathrm{s}), \mathrm{C}_{0}$ is the initial concentration over the apical side $(\mu \mathrm{g} / \mathrm{mL})$, and $\mathrm{A}$ is the surface area of monolayer $\left(\mathrm{cm}^{2}\right)$.

\section{Transport mechanism across Caco-2 cells \\ Cholesterol depletion}

Cells were first incubated with $10 \mathrm{mmol} / \mathrm{L}$ methyl- $\beta$ cyclodextrin for 1 hour at $37^{\circ} \mathrm{C} .{ }^{15,16}$ Then, a transport experiment was performed with AmB-loaded cubosomal suspension in the presence of $1 \mu \mathrm{g} / \mathrm{mL}$ lovastatin, for 2 hours at $37^{\circ} \mathrm{C}$.

\section{Inhibition of caveolin-mediated endocytosis}

Cells were first incubated with $10 \mu \mathrm{g} / \mathrm{mL}$ filipin for 1 hour at $37^{\circ} \mathrm{C},{ }^{15,16}$ and then the transport experiment was performed with AmB-loaded cubosomal suspension in the presence of $10 \mu \mathrm{g} / \mathrm{mL}$ of filipin for 2 hours at $37^{\circ} \mathrm{C}$.

\section{Inhibition of clathrin-mediated endocytosis}

Cells were first incubated with $10 \mu \mathrm{g} / \mathrm{mL}$ chlorpromazine for 1 hour at $37^{\circ} \mathrm{C}, 15,16$ and then the transport experiment was performed with AmB-loaded cubosomal suspension in the presence of $10 \mu \mathrm{g} / \mathrm{mL}$ of chlorpromazine for 2 hours at $37^{\circ} \mathrm{C}$.

\section{Inhibition of macropinocytosis}

The cells were first cultured with $550 \mathrm{mM}$ amiloride for 1 hour at $37^{\circ} \mathrm{C},{ }^{15,16}$ and then the transport experiment was performed with AmB-loaded cubosomal suspension in the presence of $550 \mathrm{mM}$ amiloride for 2 hours at $37^{\circ} \mathrm{C}$.

\section{Inhibition of P-glycoprotein (P-gP) efflux pumps}

Cells were first incubated with $100 \mathrm{mM}$ verapamil for 1 hour at $37^{\circ} \mathrm{C},{ }^{15,16}$ and then the transport experiment was performed with AmB loaded in GMO cubosomes suspension in the presence of $100 \mathrm{mM}$ verapamil for 2 hours at $37^{\circ} \mathrm{C}$.

\section{Animals}

A total of 42 male Sprague Dawley ${ }^{\circledR}$ rats weighing between 180-200 g (Guangzhou University of Chinese Medicine, Guangzhou, People's Republic of China) were used for the in vivo studies of AmB-loaded cubosomes. All rats were housed in an animal care facility under standard conditions, kept at a temperature of $22^{\circ} \mathrm{C} \pm 2{ }^{\circ} \mathrm{C}$ and relative humidity of $55 \% \pm 5 \%$. The animals were fed on a standard pellet diet, and water was made available ad libitum. Studies were approved and conducted according to the guidelines of experimental animals and the Ethical Committee of Sun Yat-sen University.

\section{Oral bioavailability studies}

The pharmacokinetic behaviors of AmB-loaded cubosomes were investigated in rats after oral administration. Twelve rats were divided randomly into two treatment groups with six rats per group as follows:

1. Group I was treated orally with Fungizone ${ }^{\circledR}$, equivalent to $10 \mathrm{mg} / \mathrm{kg} \mathrm{AmB}$.

2. Group II was treated with orally-administered AmBloaded cubosomes, equivalent to $10 \mathrm{mg} / \mathrm{kg} \mathrm{AmB}$.

Blood samples were collected into heparinized tubes at designated time intervals after administration of a single oral dose to each group, and were centrifuged at 8,000 rpm for 10 minutes. Then, $200 \mu \mathrm{L}$ of methanol:acetonitrile mixture (1:1 ratio, by volume) was added to $100 \mu \mathrm{L}$ of plasma, for precipitating the plasma proteins. AmB was extracted with the organic reagent by vigorous mixing for 1 minute. The solution was centrifuged for 10 minutes at $16,000 \mathrm{rpm}$, and $25 \mu \mathrm{L}$ of the supernatant was injected into the HPLC system, to determine the AmB content.

The HPLC method was used for analysis of AmB concentration in all samples. ${ }^{17}$ Briefly, $25 \mu \mathrm{L}$ of the extracted sample was injected into a Phenomenex Luna C-18 column (Torrance, CA, USA) at $35^{\circ} \mathrm{C}$. The wavelength of the UV detector was set at $406 \mathrm{~nm}$. A mixture of $0.01 \mathrm{M}$ phosphate buffer solution (pH: 6.2) and acetonitrile (60:40 ratio, by volume) was used as the mobile phase, at a flow rate of $0.5 \mathrm{~mL}$ per minute, for the in vivo samples.

\section{Antifungal activity in vivo}

Systemic (disseminated) candidiasis with Candida albicans ATCC $^{\circledR} 18804^{\mathrm{TM}}$ strain was developed by injecting $1 \times 10^{8}$ colony-forming units (CFU) through the tail vein. ${ }^{9}$ The infected rats were left for two days to develop a murine disseminated candidiasis model, to evaluate antifungal activity. Treatment of the infected rats started on the third day, and continued for 2 consecutive days, with AmBisome ${ }^{\circledR}$, at $5 \mathrm{mg} / \mathrm{kg}$, administered intravenously once per day; physiologic saline, administered orally (non-treated control); and AmB-loaded cubosomes at $1 \mathrm{mg} / \mathrm{kg}, 5 \mathrm{mg} / \mathrm{kg}$, and $10 \mathrm{mg} / \mathrm{kg}$, administered by oral gavage three times per day. On the fifth day, 18 hours 
after the last dose, the rats were sacrificed by inhalation of anesthetic ether. Kidney, spleen, liver, and lung were removed aseptically, and placed in a tissue homogenizer with sterile saline solution (1:2 ratio, tissue:saline). The number of CFUs in the organs was determined by a plate dilution method. Ten-fold serial dilutions of $0.1 \mathrm{~mL}$ of homogenate were plated onto duplicate Saboraud Dextrose Agar plates, then incubated for 48 hours at $37^{\circ} \mathrm{C}$. Finally, the resulting colonies of $C$. albicans were counted and averaged over the duplicate plates. The course of $C$. albicans infections was monitored by evaluating the fungal load in these organs.

\section{Statistics}

Pharmacokinetic parameters were determined by using 3p97 computer software (Chinese Association of Mathematical Pharmacology, Beijing, People's Republic of China). Statistical significance in the difference of the means was evaluated by using the Student's $t$-test.

\section{Results \\ Cubic structure of AmB loaded in GMO cubosomes}

The average diameter of the cubosomes was $192.3 \pm 10.8 \mathrm{~nm}$, with a polydispersity index of $0.20 \pm 0.02$, indicating a uniform size distribution (Figure 1).

\section{AmB incorporated in cubosomes}

The centrifugal ultrafiltration method demonstrated that the AmB encapsulation efficiency in cubosomal formulation reached $94.0 \%$, showing that more than $90 \%$ AmB was successfully encapsulated in GMO cubosomes.

Furthermore, our group demonstrated the encapsulation of AmB loaded in cubosomes through UV-visible spectrum analysis. As shown in Figure 2, the major absorption bands, at $328 \mathrm{~nm}$ and $409 \mathrm{~nm}$, were found in both the free AmB solution and the physical mixture of AmB and blank cubosomes, whereas the spectroscopy of AmB-loaded cubosomes showed an absorption peak at the same position, with much lower intensity. Therefore, these UV-visible spectrum results further confirmed that $\mathrm{AmB}$ was actually encapsulated in GMO cubosomes, not simply mixed with blank cubosomes.

\section{Aggregation state in SGF or FASSIF}

As shown in Figure 3, some changes among AmB deoxycholate, AmB-loaded cubosomes, and a mixture of AmB deoxycholate and blank cubosomes were clearly observed after SGF incubation for 30 minutes. AmB aggregation was found in both $\mathrm{AmB}$ solution and the mixture of AmB and blank cubosomes, while a dispersed state was clearly observed in AmB-loaded cubosomes.

\section{Transport study across Caco- 2 cells}

As seen in Figure 4, A-B transport was effective in the following order: AmB-loaded cubosomes $>$ Fungizone $^{\circledR}$. B-A transport was in the same order: AmB-loaded cubosomes $>$ Fungizone $^{\circledR}$. The transport profile of AmBloaded cubosomes increased 5.1 times, compared against that of Fungizone ${ }^{\circledR}$ in A to B transport $\left(182.7 \mathrm{ng} / \mathrm{cm}^{2}\right.$ versus $\left.35.5 \mathrm{ng} / \mathrm{cm}^{2} ; P<0.01\right)$. Moreover, AmB-loaded cubosomes in B-A transport still showed a significantly higher transport profile $\left(50.3 \mathrm{ng} / \mathrm{cm}^{2}\right)$, compared against that of Fungizone ${ }^{\circledR}$ $\left(195.6 \mathrm{ng} / \mathrm{cm}^{2}\right)$. This suggested that the transport profiles of cubosomes were significantly higher than that of Fungizone ${ }^{\circledR}$ in the transport study, across the Caco-2 cell monolayer (Table 1).

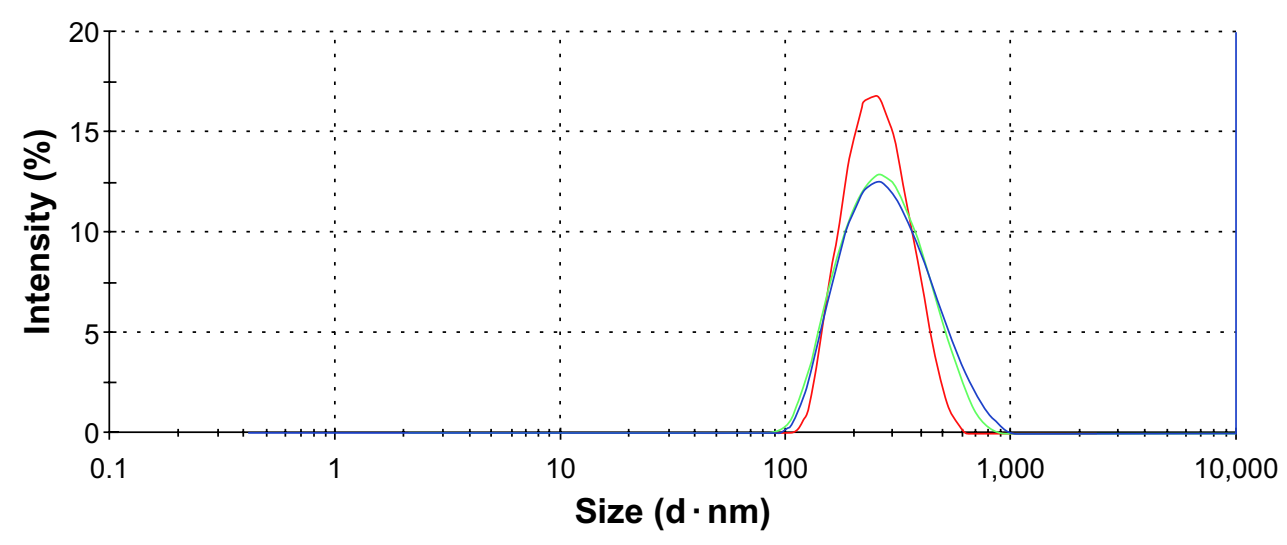

Figure I Particle size distribution of cubosome dispersion, measured three times via Zetasizer Nano ZS90. Note: Each data point represents the mean \pm standard deviation of three determinations. 

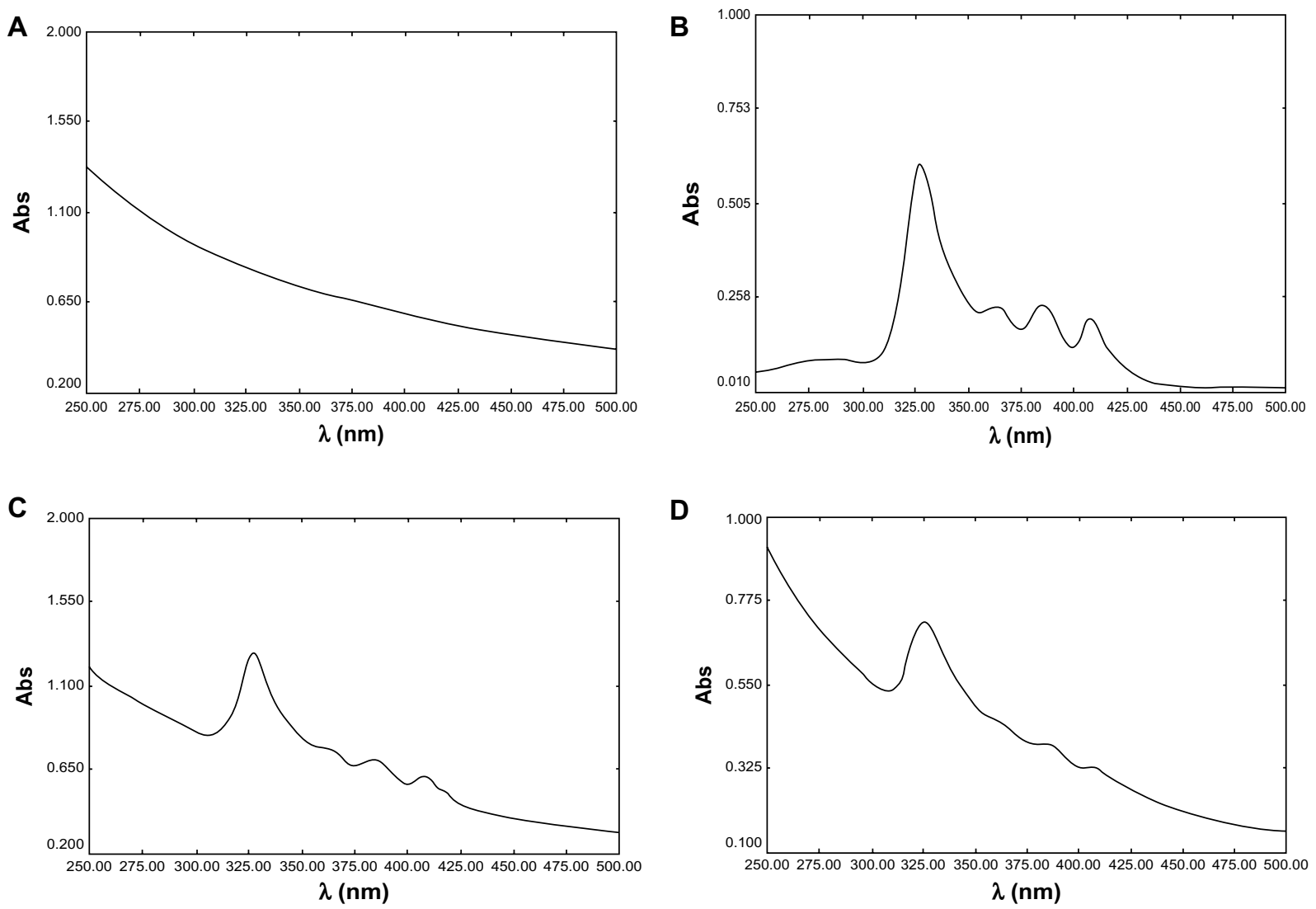

Figure 2 Ultraviolet spectra of AmB: (A) blank cubosomes; (B) AmB deoxycholate; (C) mixture of AmB deoxycholate and blank cubosomes; (D) AmB-loaded cubosomes. Abbreviations: AmB, amphotericin B; Abs, absorbance.

\section{Transport mechanism across Caco-2 cells}

In the transport study, there was about a $10 \%-20 \%$ reduction on the TEER values of Caco-2 monolayers. Because a study on labrasol revealed that up to $80 \%$ of
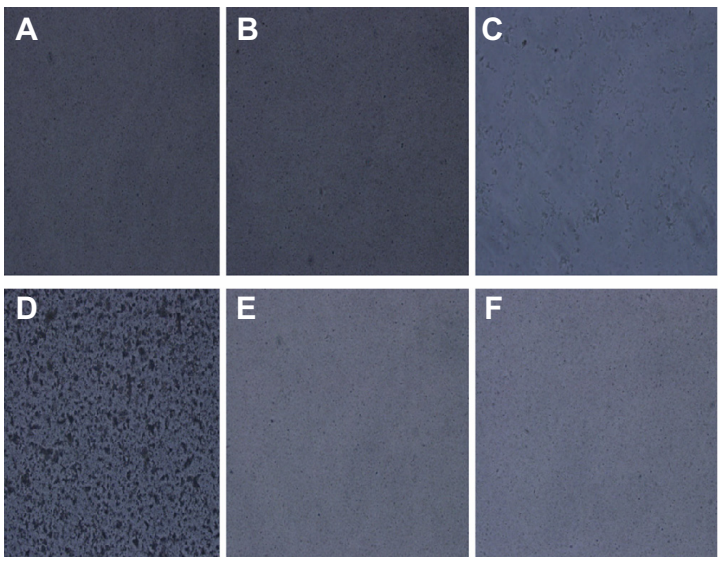

Figure 3 Optical microscopy $(\times 200)$ of samples: (A) AmB-loaded cubosomes in SGF; (B) blank GMO cubosomes in SGF; (C) AmB deoxycholate in SGF; (D) mixture of AmB deoxycholate and blank cubosomes in SGF; (E) AmB-loaded cubosomes in FASSIF; (F) blank GMO cubosomes in FASSIF.

Abbreviations: AmB, amphotericin B; SGF, simulated gastric fluid; GMO, glyceryl monoolein; FASSIF, fasting state simulated intestinal fluid.
A

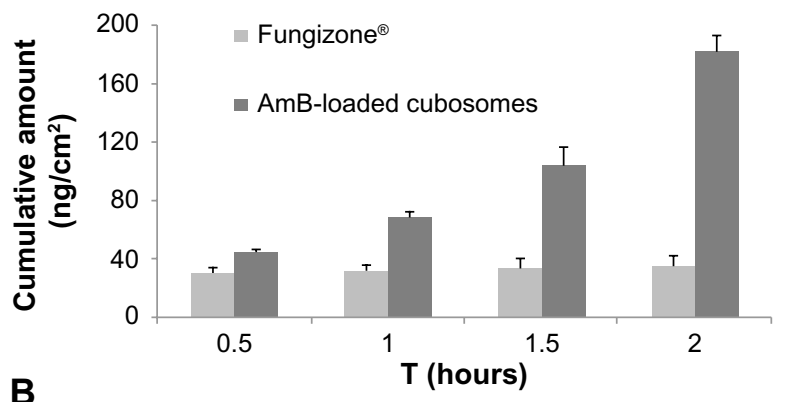

B

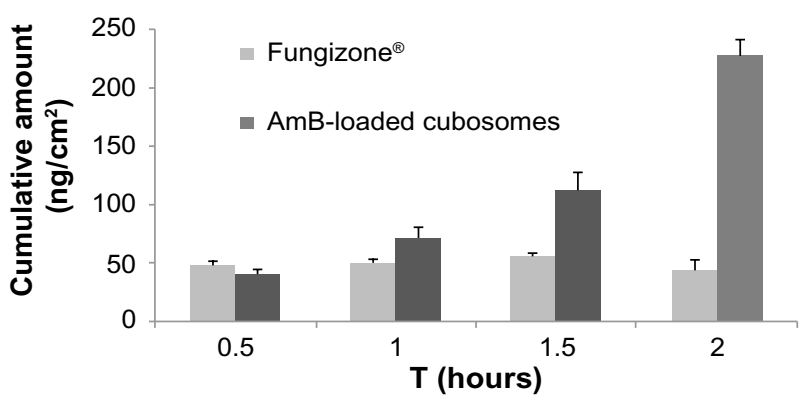

Figure 4 Transport profiles of AmB from Fungizone ${ }^{\circledR}$, and AmB-loaded cubosomes, across the Caco-2 cell monolayer: (A) apical to basolateral; (B) basolateral to apical.

Note: Each data point represents the mean \pm standard deviation of three determinations.

Abbreviations: AmB, amphotericin B; Caco-2, human colon adenocarcinoma cell line. 
Table I The apparent permeabilities of AmB across Caco- 2 cell monolayer from the two formulations

\begin{tabular}{lll}
\hline Formulation & $\begin{array}{l}\text { Permeability coefficient } \\
\left(\times 10^{-5} \mathrm{~cm} / \mathrm{s}\right)\end{array}$ \\
\cline { 2 - 3 } & A to B & B to A \\
\hline Fungizone $^{\circledR}$ & $0.10 \pm 0.02$ & $0.18 \pm 0.03$ \\
AmB-loaded cubosomes & $0.50 \pm 0.12$ & $0.58 \pm 0.15$ \\
\hline
\end{tabular}

Note: Each data point represents the mean \pm standard deviation of three determinations.

Abbreviations: AmB, amphotericin B; Caco-2, human colon adenocarcinoma cell line; A to B, apical to basolateral; B to A, basolateral to apical.

TEER value reduction could disrupt the tight junction, ${ }^{18}$ it could be concluded that GMO cubosomes did not show any ability to alter the integrity of the Caco-2 cellular tight junctions.

As seen in Figure 5, about a 4.2\% reduction of AmB transport was observed under the conditions of cholesterol depletion. This result indicated that no difference was observed in the internalized quantities of $\mathrm{AmB}$ in the cholesterol inhibition experiment. Second, incubation of Caco-2 cells with GMO cubosomes in the presence of filipin was performed to disrupt the caveolae structure, by binding to sterols, such as cholesterol, and disorganizing the caveolin. ${ }^{19}$ The results showed about a $38.8 \%$ reduction of AmB, compared against the uptake of the control group, suggesting the Caco-2 uptake of AmB-loaded cubosomes occurred through caveolin-mediated endocytosis. Third, the presence of chlorpromazine, a clathrin inhibitor, was used to reduce the number of pit-associated receptors at the cell surface, by disrupting the assembly and disassembly of clathrin. A decrease in the transport of AmB-loaded cubosomes was also found, but it was less pronounced than that found with filipin. Moreover, experiments were performed in the presence of amiloride, to investigate macropinocytosis. The presence of amiloride, a macropinocytosis inhibitor, had no effect on the uptake of AmB-loaded cubosomes, implying

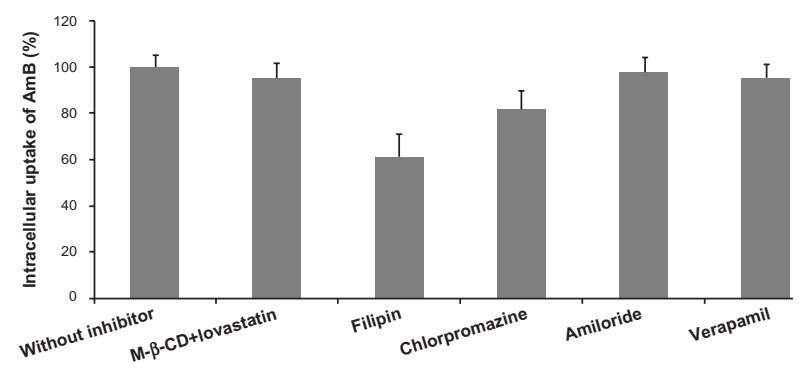

Figure 5 Variation of intracellular uptake of AmB after 2 hours of incubation with AmB-loaded cubosomes and different endocytosis inhibitors.

Note: Each data point represents the mean \pm standard deviation of three determinations.

Abbreviation: AmB, amphotericin B. that the endocytosis of AmB-loaded cubosomes did not occur via macropinocytosis. Finally, verapamil, a traditional P-gp inhibitor, was used to study the potential effect of cubosomes on P-gp efflux pump inhibition. No significant difference was observed between the Caco-2 uptake of AmB-loaded cubosomes, with and without verapamil, implying that AmBloaded cubosomes were not internalized by Caco-2 cells in a P-gp-independent way.

\section{Pharmacokinetic studies}

Figure 6 shows the plasma concentration profiles of AmB after single-dose oral administration of the two formulations to rats. The major pharmacokinetic parameters, including $\mathrm{C}_{\max }$ (maximum drug concentration), $\mathrm{T}_{\max }$ (time to maximum plasma concentration), and area under the curve (AUC) are summarized in Table 2.

The plasma concentration of Fungizone ${ }^{\circledR}$ could be detected for only 2 days, whereas the plasma drug profile of AmB-loaded cubosomal formulations showed a sustained release of AmB over a period of 4 days. Furthermore, AmBloaded cubosomes led to higher $\mathrm{C}_{\max }(286.4 \pm 35.1 \mathrm{ng} / \mathrm{mL})$ and AUC $(11,749.1 \pm 2,231.2 \mathrm{~h} \cdot \mathrm{ng} / \mathrm{mL})$ values.

\section{Antifungal activity in vivo}

Figure 7 shows that, following treatment with IV AmBisome ${ }^{\circledR}$, C. albicans infection of the harvested organs was significantly lower than in the untreated control group of rats. Treatment with orally-administered, AmB-loaded cubosomes significantly reduced the fungal burden, and showed a dose-dependent response in kidney tissues, compared against the untreated group. Oral administration of AmB-loaded cubosomes at doses of $10 \mathrm{mg} / \mathrm{kg}, 5 \mathrm{mg} / \mathrm{kg}$, and $1 \mathrm{mg} / \mathrm{kg}$ led to fungal reductions of $90.7 \%, 67.3 \%$, and $39.6 \%$, respectively. In the lung, liver and, spleen tissues, the fungal burden showed no significant reduction, even at the highest dose of $10 \mathrm{mg} / \mathrm{kg}$, compared against the untreated group.

\section{Discussion}

The stability of nanocarriers in the gastrointestinal tract plays a major role in determining the rate and extent of absorption of drugs from the tract. Nguyen et al observed that the effect of enzymatic degradation on the internal phase structure of GMO cubosomes was determined, over time, using smallangle X-ray scattering. ${ }^{12}$ It was observed that lipolytic and/or acid-catalyzed degradation of GMO led to a loss of liquid crystalline structure of the cubosomes, suggesting that the fasted gastrointestinal environment was reduced, to some extent, by the phase structure of GMO cubosomes. ${ }^{12}$ 


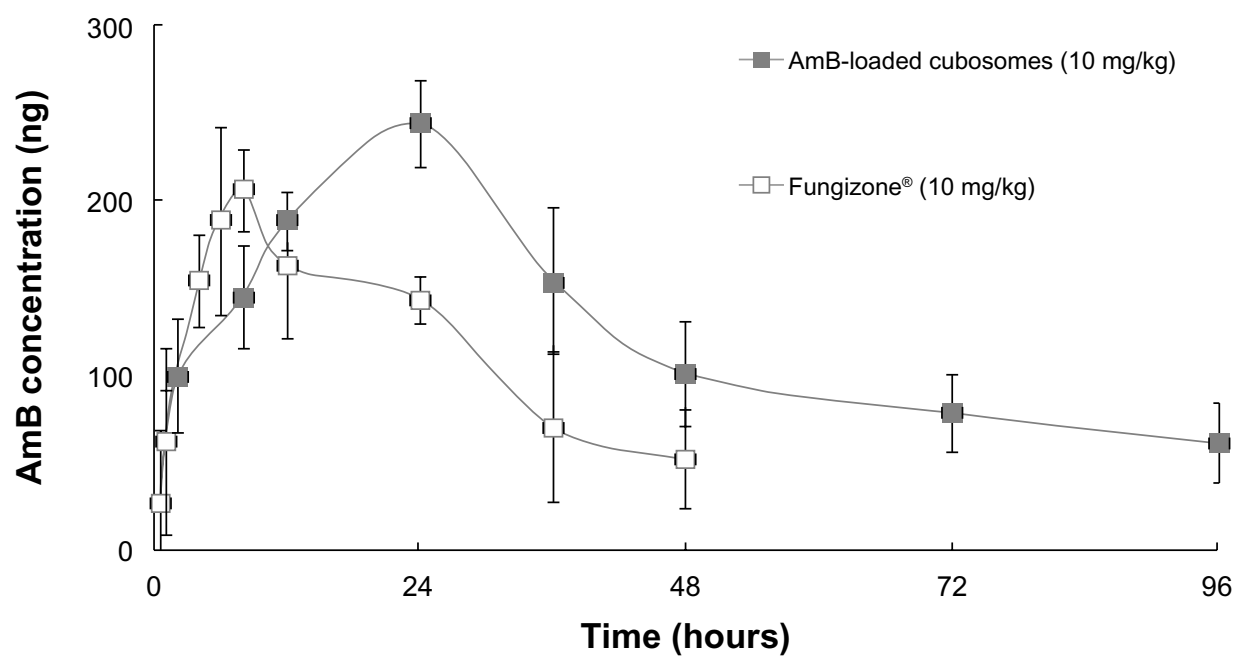

Figure 6 Comparative in vivo plasma concentration versus time profiles, following a single, orally-administered dose.

Note: Each data point represents the mean \pm standard deviation of six determinations.

Abbreviation: AmB, amphotericin B.

After meeting the first barrier faced by GMO cubosomes in the gastrointestinal environment, Caco-2 cell monolayers were used to determine the transport mechanism of GMO cubosomes in an intestinal cell culture model. Two possible uptake mechanisms can be suggested for oral absorption of nanoparticles: ${ }^{20,21}$ a paracellular transport pathway, via the tight junctions, and a transcellular transport pathway, via the intestinal barrier.

Paracellular transport is passive diffusion through intercellular spaces. Tight junctions are closely-associated areas of two cells that permit the formation of almost impermeable barriers, open only to allow small molecules to pass. Consequently, paracellular transport between the epithelial cells is controlled by the size of the intercellular space, whose pore diameter has been estimated to be between 3-10 $\AA^{22,23}$ To allow drug passage, tight junctions need to be opened. In the present experiment, there was no reduction of the TEER values of Caco-2 monolayers. As a result, the integrity of Caco-2 cell monolayers was preserved, indicating

Table 2 Pharmacokinetic parameters upon oral administration of Fungizone ${ }^{\circledR}$ and AmB-loaded cubosomes

\begin{tabular}{lllll}
\hline Formulation & $\begin{array}{l}\mathbf{C}_{\max } \\
(\mathbf{n g} / \mathbf{m L})\end{array}$ & $\mathbf{T}_{\max }(\mathbf{h})$ & $\mathbf{t}_{1 / 2}(\mathbf{h})$ & $\begin{array}{l}\mathbf{A U C}_{0-120} \\
(\mathbf{h} \cdot \mathbf{n g} / \mathbf{m L})\end{array}$ \\
\hline $\begin{array}{l}\text { Fungizone }^{\circledR} \\
(10 \mathrm{mg} / \mathrm{kg})\end{array}$ & $218.5 \pm 30.9$ & $6.3 \pm 2.3$ & $21.4 \pm 6.1$ & $5,964.0 \pm 705.4$ \\
$\begin{array}{l}\text { AmB-loaded } \\
\text { cubosomes } \\
(10 \mathrm{mg} / \mathrm{kg})\end{array}$ & $286.4 \pm 35.1$ & $25.1 \pm 3.7$ & $20.2 \pm 5.7$ & $11,749.1 \pm 2,231.2$ \\
\hline
\end{tabular}

Note: Each data point represents the mean \pm standard deviation of three determinations.

Abbreviation: $A m B$, amphotericin $B ; C_{\max }$, maximum drug concentration; $T_{\max }$, time to maximum plasma concentration; $\mathrm{t}_{1 / 2}$, terminal phase half-life; $\mathrm{AUC}_{0-120}$, area under the curve (0-120 minutes). that the tight junctions were not altered in the presence of GMO cubosomes. That there was no opening of intercellular tight junctions could be explained by the fact that the negatively charged particles had no effect on disrupting the tight junctions. ${ }^{24}$

After having demonstrated that GMO cubosomes did not route through the paracellular pathway, energydependent transcellular transport of GMO cubosomes into Caco-2 cells should be considered. Uptake by enterocytes is described by pinocytosis, including macropinocytosis or endocytosis, which includes clathrin-mediated endocytosis, caveolae-mediated endocytosis, and clathrin- and caveolaeindependent endocytosis. ${ }^{25-27}$

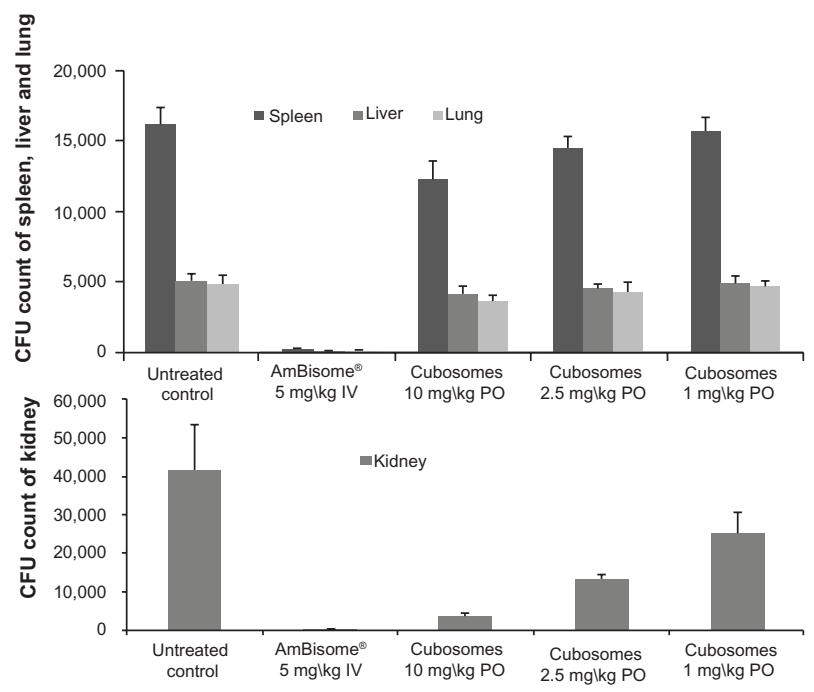

Figure 7 Comparison of the efficacy of oral AmB loaded in cubosomal formulation with treatment of IV AmBisome ${ }^{\circledR}$ in the kidneys, spleen, liver, and lungs of a rat model of invasive candidiasis.

Abbreviations: AmB, amphotericin B; IV, intravenous; PO, oral administration; CFU, colony forming units. 
A difference in $\mathrm{AmB}$ transport was not observed in the cholesterol inhibition, macropinocytosis inhibition, and P-gp inhibition experiments. Transport of AmB loaded in GMO cubosomes showed a significant decrease after the administration of filipin or chlorpromazine. Thus, the Caco-2 uptake of AmB loaded in GMO cubosomes was through clathrin- and caveolin-mediated endocytosis.

In the transport study, GMO cubosomes exhibited a higher amount of A-B transport of of AmB than did Fungizone ${ }^{\circledR}$. Its inhibiting capacity may result from the P-gp inhibition of GMO. As reported in the literature, Peceol ${ }^{\circledR}$ could significantly increase the gastrointestinal absorption of AmB. The enhancement of absorption contributed to a decrease in the expression of multidrug resistance polypeptide $1 \mathrm{mRNA}$, and $\mathrm{P}$-gp protein, resulting in lower P-gp-mediated AmB efflux. ${ }^{28}$ However, this is not direct evidence that $\mathrm{AmB}$ is a P-gp substrate, and that the oral absorption is affected by P-gp inhibition. Thus, significant work still needs to be done to obtain an in-depth understanding of the absorption mechanism of GMO cubosomes.

Cubosomes are bicontinuous cubic phase liquid crystals, with high surface area and short diffusion distance for releasing drugs from the cubosomes. It is expected that drugs loaded into cubosomes would release rapidly under sink conditions. ${ }^{12,29}$ In addition, GMO, with an ester-based structure, may be rapidly cleaved by pancreatic lipase and ester hydrolysis in the gastrointestinal fluids. Furthermore, $\mathrm{AmB}$ has very low aqueous solubility, but high solubility in the acidic environment of gastric fluid. Thus, AmB loaded in GMO cubosomes was significantly reduced, due to the rapid release of AmB from GMO cubosomes in the stomach.

At this phase, the drug released from the formulation, due to either precipitation or dissolution into the gastrointestinal media, is resolubilized as micelles, or mixed micelles, by emulsifications. Both of these can play a significant role in the performance of the formulation. ${ }^{30}$ The overall in vivo solubilization capacity depends on the lipophilicity, the chemical structure of the drug, and the nature of the endogenous and exogenous lipids involved in forming colloidal species. ${ }^{31}$ It had been shown that GMO emulsion was capable of stimulating an increase in the absorption of $\mathrm{AmB}$ in Sprague Dawley rats. ${ }^{28,32}$ This result could be explained by the fact that the degradation of GMO emulsion led to the transporting of AmB into the circulatory system, in the form of either micelles or mixed micelles. In view of the fact that $\mathrm{AmB}$ was released rapidly from cubosomes, and that GMO cubosomes were degraded in the gastrointestinal tract, $\mathrm{AmB}$ released from GMO cubosomes into the aqueous layer was solubilized in micellar structures, which were ready for immediate absorption.

AmB released from GMO cubosomes into the aqueous layer can be absorbed at the early stage, due to the micelle form, to overcome the disadvantage of GMO degradation. By contrast, AmB loaded in cubosomes can be absorbed at the later stage, due to the slow release from cubosomes. As expected, the AUC value for oral administration of $\mathrm{AmB}$ loaded in GMO cubosomes was higher than for administration of oral Fungizone ${ }^{\circledR}$, even equivalent to that of $\mathrm{AmB}$ loaded in PHY cubosomes. ${ }^{6}$ The study also pointed out that the poor absorption of Fungizone ${ }^{\circledR}$ could contribute to the aggregation state found in SGF, despite that Fungizone ${ }^{\circledR}$ was made into a micellar system.

The concentration of the therapeutic plasma drug in patients after oral administration is a serious concern. However, no standard is currently available. After AmB loaded in GMO cubosomes were administered to rats, antifungal efficacy was assessed by the reduction of fungal burden expressed (number of CFUs in various organs), compared against an untreated control group. ${ }^{33}$ The efficacy of the oral GMO cubosomal formulation was similar to that of the previous AmB emulsion (reported by Sivak et al, in Canada). ${ }^{34}$ A linear correlation was found between antifungal activity in the kidneys and increase of $\mathrm{AmB}$ levels in renal tissues, following the administration of a GMO cubosomal formulation. Consistent with a previous study, ${ }^{34}$ low levels of AmB, following the administration of GMO cubosomes, reached therapeutic concentrations in the kidneys to show comparable efficacy to AmBisome ${ }^{\circledR}$. This study showed a shift in distribution to the kidneys, after oral administration of GMO cubosomes. The dose of AmB-loaded cubosomes may play a key role in the kidney distribution, as reported in PHY cubosomes and emulsion. ${ }^{6,35}$ A possible reason for the kidney distribution of AmB-loaded cubosomes need to be elucidated in future research. Furthermore, in view of unique, multilayer cubosomal structures and bicontinuous cubic phase in excess water, GMO cubosomes are more stable, in comparison to emulsion. Thus, the results obtained from the present study suggest that GMO cubosomes, as a safe lipid formulation, provide a better alternative for oral administration of AmB.

\section{Conclusion}

The proposed regimen for oral GMO cubosomes was successful in reducing the fungal burden in the kidney, which indicated an effective approach for enhancing the oral bioavailability of AmB. In addition, GMO cubosomes were taken up by Caco-2 cells, mainly via clathrin-dependent and caveolae-dependent transport mechanisms. 


\section{Acknowledgments}

The authors are grateful to BASF Company, Germany, for generous gift samples of Poloxamer 407. We thank the Nanjing Medical University for their financial support (2012NJMU243).

\section{Disclosure}

The authors report no conflicts of interest in this work.

\section{References}

1. Benincasa M, Pacor S, Wu W, Prato M, Bianco A, Gennaro R. Antifungal activity of amphotericin B conjugated to carbon nanotubes. ACS Nano. 2011;5(1):199-208.

2. Jain S, Valvi PU, Swarnakar NK, Thanki K. Gelatin coated hybrid lipid nanoparticles for oral delivery of amphotericin B. Mol Pharm. 2012;9(9):2542-2553.

3. Verma RK, Pandya S, Misra A. Loading and release of amphotericin-B from biodegradable poly(lactic-co-glycolic acid) nanoparticles. J Biomed Nanotechnol. 2011;7(1):118-120.

4. Golenser J, Domb A. New formulations and derivatives of amphotericin B for treatment of leishmaniasis. Mini Rev Med Chem. 2006;6(2):153-162.

5. Patel PA, Patravale VB. AmbiOnp: solid lipid nanoparticles of amphotericin B for oral administration. J Biomed Nanotechnol. 2011;7(5):632-639.

6. Yang Z, Tan Y, Chen M, et al. Development of amphotericin B-loaded cubosomes through the SolEmuls technology for enhancing the oral bioavailability. AAPS PharmSciTech. 2012;13(4):1483-1491.

7. Yang Z, Peng X, Tan Y, et al. Optimization of the preparation process for an oral phytantriol-based amphotericin B cubosomes. J Nanomater. 2011 2011: Article ID 308016.

8. Wasan EK, Bartlett K, Gershkovich P, et al. Development and characterization of oral lipid-based amphotericin B formulations with enhanced drug solubility, stability and antifungal activity in rats infected with Aspergillus fumigatus or Candida albicans. Int J Pharm. 2009;372(1-2):76-84.

9. Ibrahim F, Gershkovich P, Sivak O, Wasan EK, Bartlett K, Wasan KM. Efficacy and toxicity of a tropically stable lipid-based formulation of amphotericin B (iCo-010) in a rat model of invasive candidiasis. Int $J$ Pharm. 2012;436(1-2):318-323.

10. Perlin DS. Amphotericin B cochleates: a vehicle for oral delivery. Curr Opin Investig Drugs. 2004;5(2):198-201.

11. Thornton SJ, Wasan KM. The reformulation of amphotericin B for oral administration to treat systemic fungal infections and visceral leishmaniasis. Expert Opin Drug Deliv. 2009;6(3):271-284.

12. Nguyen TH, Hanley T, Porter CJ, Boyd BJ. Nanostructured liquid crystalline particles provide long duration sustained-release effect for a poorly water soluble drug after oral administration. J Control Release. 2011;153(2):180-186.

13. Zhen G, Hinton TM, Muir BW, et al. Glycerol monooleate-based nanocarriers for siRNA delivery in vitro. Mol Pharm. 2012;9(9):2450-2457.

14. Hosmer JM, Steiner AA, Lopes LB. Lamellar liquid crystalline phases for cutaneous delivery of paclitaxel: Impact of the monoglyceride. Pharm Res. 2012;30(3):694-706.

15. Roger E, Lagarce F, Garcion E, Benoit JP. Lipid nanocarriers improve paclitaxel transport throughout human intestinal epithelial cells by using vesicle-mediated transcytosis. J Control Release. 2009;140(2): 174-181.

16. Mo R, Jin X, Li N, et al. The mechanism of enhancement on oral absorption of paclitaxel by N-octyl-O-sulfate chitosan micelles. Biomaterials. 2011;32(20):4609-4620.
17. Robbie G, Chiou WL. Elucidation of human amphotericin B pharmacokinetics: identification of a new potential factor affecting interspecies pharmacokinetic scaling. Pharm Res. 1998;15(10):1630-1636.

18. Li N, Li XR, Zhou YX, et al. The use of polyion complex micelles to enhance the oral delivery of salmon calcitonin and transport mechanism across the intestinal epithelial barrier. Biomaterials. 2012;33(34): 8881-8892.

19. Vercauteren D, Rejman J, Martens TF, Demeester J, De Smedt SC, Braeckmans K. On the cellular processing of non-viral nanomedicines for nucleic acid delivery: mechanisms and methods. J Control Release. 2012;161(2):566-581.

20. Incecayir T, Tsume Y, Amidon GL. Comparison of the permeability of metoprolol and labetalol in rat, mouse, and Caco-2 cells: use as a reference standard for BCS classification. Mol Pharm. 2013;10(3): 958-966.

21. Frolund S, Langthaler L, Kall MA, Holm R, Nielsen CU. Intestinal drug transport via the proton-coupled amino acid transporter PAT1 (SLC36A1) is inhibited by Gly-X(aa) dipeptides. Mol Pharm. 2012;9(9):2761-2769.

22. Jahn MR, Nawroth T, Futterer S, Wolfrum U, Kolb U, Langguth P. Iron oxide/hydroxide nanoparticles with negatively charged shells show increased uptake in Caco-2 cells. Mol Pharm. 2012;9(6):1628-1637.

23. Roger E, Kalscheuer S, Kirtane A, et al. Folic acid functionalized nanoparticles for enhanced oral drug delivery. Mol Pharm. June 13, 2012. [Epub ahead of print.]

24. Damge C, Reis CP, Maincent P. Nanoparticle strategies for the oral delivery of insulin. Expert Opin Drug Deliv. 2008;5(1):45-68.

25. Lin YH, Mi FL, Chen CT, et al. Preparation and characterization of nanoparticles shelled with chitosan for oral insulin delivery. Biomacromolecules. 2007;8(1):146-152.

26. Vertzoni M, Markopoulos C, Symillides M, Goumas C, Imanidis G, Reppas C. Luminal lipid phases after administration of a triglyceride solution of danazol in the fed state and their contribution to the flux of danazol across Caco-2 cell monolayers. Mol Pharm. 2012;9(5):1189-1198.

27. Meng S, Wu B, Singh R, et al. SULT1A3-mediated regiospecific 7-O-sulfation of flavonoids in Caco- 2 cells can be explained by the relevant molecular docking studies. Mol Pharm. 2012;9(4):862-873.

28. Risovic V, Sachs-Barrable K, Boyd M, Wasan KM. Potential mechanisms by which Peceol increases the gastrointestinal absorption of amphotericin B. Drug Dev Ind Pharm. 2004;30(7):767-774.

29. Rosenblatt KM, Douroumis D, Bunjes H. Drug release from differently structured monoolein/poloxamer nanodispersions studied with differential pulse polarography and ultrafiltration at low pressure. J Pharm Sci. 2007;96(6):1564-1575.

30. Fatouros DG, Bergenstahl B, Mullertz A. Morphological observations on a lipid-based drug delivery system during in vitro digestion. Eur J Pharm Sci. 2007;31(2):85-94.

31. Kossena GA, Charman WN, Wilson CG, et al. Low dose lipid formulations: effects on gastric emptying and biliary secretion. Pharm Res. 2007;24(11):2084-2096.

32. Sachs-Barrable K, Lee SD, Wasan EK, Thornton SJ, Wasan KM. Enhancing drug absorption using lipids: a case study presenting the development and pharmacological evaluation of a novel lipid-based oral amphotericin B formulation for the treatment of systemic fungal infections. Adv Drug Deliv Rev. 2008;60(6):692-701.

33. Risovic V, Boyd M, Choo E, Wasan KM. Effects of lipid-based oral formulations on plasma and tissue amphotericin B concentrations and renal toxicity in male rats. Antimicrob Agents Chemother. 2003;47(10): 3339-3342.

34. Sivak O, Gershkovich P, Lin M, et al. Tropically stable novel oral lipid formulation of amphotericin $\mathrm{B}$ (iCo-010): biodistribution and toxicity in a mouse model. Lipids Health Dis. 2011;10:135.

35. Gershkovich P, Wasan EK, Lin M, et al. Pharmacokinetics and biodistribution of amphotericin B in rats following oral administration in a novel lipid-based formulation. JAntimicrob Chemother. 2009;64(1): $101-108$. 


\section{Publish your work in this journal}

The International Journal of Nanomedicine is an international, peerreviewed journal focusing on the application of nanotechnology in diagnostics, therapeutics, and drug delivery systems throughout the biomedical field. This journal is indexed on PubMed Central, MedLine, CAS, SciSearch ${ }^{\circledR}$, Current Contents ${ }^{\circledR} /$ Clinical Medicine,

Journal Citation Reports/Science Edition, EMBase, Scopus and the Elsevier Bibliographic databases. The manuscript management system is completely online and includes a very quick and fair peer-review system, which is all easy to use. Visit http://www.dovepress.com/ testimonials.php to read real quotes from published authors.

Submit your manuscript here: http://www.dovepress.com/international-journal-of-nanomedicine-journal 